\title{
Physical Activity Prescription for Non-Communicable Diseases: Practices of Healthcare Professionals in Hospital Setting, Ethiopia.
}

\author{
Getu Teferi ${ }^{1}$,Harish Kumar $^{2}$,Paramvir Singh ${ }^{3}$ \\ ${ }^{I}$ ph.D Student In Faculty Of Medicine, Department Of Sports Science, Punjabi University, \\ Patiala, India \\ ${ }^{2}$ Assistant Professor, Department Of Sports Science, Punjabi University, Patiala, India \\ ${ }^{3}$ Professor \& Head, Department Of Sports Science, Director ETI \& Prog. Cord. NSS Punjabi \\ University, Patiala, India
}

\begin{abstract}
Introduction: Regular physical activity ( PA) plays an important role in the primary and secondary prevention of several chronic diseases, e.g., cardiovascular disease, diabetes, cancer, hypertension, obesity, depression and osteoporosis (WHO 2010, ACSM 2009). Here the healthcare setting has been recognized as an appropriate and promising venue for counseling and prescribing physical activity (Jacobson et al; 2005).

Methods and materials: A cross-sectional survey design was used to assess healthcare professionals' physical activity counseling and prescription practice for non-communicable diseases in hospital setting. The sample hospitals were selected randomly based on the inclusion and exclusion criteria. The sample size was determined by using the formula for estimating a single population proportion.

Results: A total of 442 healthcare professionals from 7 government hospitals in Addis Ababa city were participated in the study. From these hospitals 387 healthcare professionals (physicians $=135,34.9 \%$, nurses $=$ $218,56.3 \%$ and physiotherapist $=34,8.8 \%)$ were completed the questionnaire. Healthcare professionals (HCPs) reported that, they very frequently ask patients about their physical activity level (8.8\%), assess patients' fitness as part of a physical exam or through a fitness test (2.3\%), Refer patients to other professionals for fitness assessment or appraisal (5.2\%), Provide patients with verbal directions for a physical activity program (21.4 $\%)$, provide patients with written directions for a physical activity program (5.4\%), counsel PA for the purpose of preventing chronic diseases (9.2\%) and Counsel PA for the purpose of treating chronic diseases $(5.5 \%)$. Personal PA level was positively and significantly correlated with PA prescription/counseling practices $\left(r_{s}=\right.$ $.17, p=.00)$. There were a significant difference among the three groups of $\operatorname{HCPs}\left(\chi^{2}=147.85, d f=2, p=\right.$ $.000)$.

Conclusion: Healthcare professionals' PA prescription/counseling rates was low in hospital setting. The need for improved implementation and establish strategies to increases HCPs' practice of PA prescription/counseling in hospital setting.
\end{abstract}

Keywords: Physical activity prescription/counseling, Non-communicable diseases, Healthcare professionals, Healthcare setting, Practice and Barriers

\section{Introduction}

Physical activity counseling and prescription have been discussed extensively for the last decade as a means for preventing or treating non-communicable diseases (NCDs) in healthcare settings. Studies show that the majority of patients listen to their physicians with regard to lifestyle behavior guidance, such as diet and physical activity. Still, commitment to follow physical activity counseling and the motivation to comply with physical activity prescriptions are major challenges faced by patients and the healthcare professionals (Daley, 2008). The health benefits of physical activity (PA) are well known, and PA is fundamental to energy balance and weight control. Regular PA plays an important role in the primary and secondary prevention of several chronic diseases, e.g., cardiovascular disease, diabetes, cancer, hypertension, obesity, depression and osteoporosis (WHO 2010, ACSM 2009). Physical inactivity has been identified as the fourth leading risk factor for global mortality, accounting for $9 \%$ of global deaths or 5.3 million of deaths globally in 2008 (Lee I et al., 2012; World Health Organization, 2009). A physically inactive lifestyle increases all-cause mortality risk by 20$30 \%$ compared to sufficiently physically active people (Lee \& Skerrett, 2001; Physical Activity Guidelines Advisory Committee, 2008)

The main focus of health care for NCDs in many low- and middle-income countries is hospital centered acute care. NCD patients present at hospitals when cardiovascular disease, cancer, diabetes and chronic respiratory disease have reached the point of acute events or long-term complications. This is a very expensive 
approach that will not contribute to a significant reduction of the NCD burden (WHO, 2011). In other hand establishing a strategy of physical activity prescription/counseling by healthcare professionals in healthcare setting for the purpose of preventing and managing chronic diseases is very important. In line with this, regular physical activity protects against chronic diseases (Lee et al., 2012). Further, regular physical activity can achieve parallel or greater effects on NCDs risk factors than those achieved with drugs at a lower cost and with minimal adverse effects (Fiuza-Luces, Garatachea, Berger, \& Lucia, 2013). Thus, increasing physical activity at the population level has become an essential component of major global initiatives to improve health (WHO, 2012). Approaches to promote increased levels of PA for patients, vary from simple oral advice, "to be a little more active", to more structural counseling. The most structured PA counseling uses established behavioral strategies to change the lifestyle behaviour of the individual. PA could also be delivered as part of a exercise referral scheme, but also as part of the in-hospital services (Borjesson M.; 2013).

The healthcare setting has been recognized as an appropriate and promising venue for counseling and prescribing physical activity (Jacobson et al; 2005). In developing countries, where healthcare professionals hold a respected position, this setting may exert a strong influence on patients' behaviours (Holub et al., 2013). Physical activity can be effectively applied in practice for disease prevention and treatment in healthcare settings (Meyer et al., 2010). Vuori, Lavie and Blair (2013) suggested that especially brief PA prescription/counseling is an efficient, effective, and cost-effective means to increase PA to bring considerable clinical benefits to various patients. Furthermore, it can be practiced as part of the routine work of the healthcare professionals (HCPs). However, there is a need and feasible means to increase the use and improve the quality of PA counseling.

Over the past decade, practitioners in health-care (HC) settings in many countries have promoted increased physical activity or exercise through written prescriptions or verbal counseling (Elley, et al;2003 and Kalling, et al; 2008). But in Ethiopia there is no clear evidence and strategy to prevent and manage NCDs through physical activity. Therefore the purpose of this study is to assess healthcare professionals' PA prescription/counseling practices among healthcare professionals in hospital setting.

\subsection{Research Design}

\section{Materials and Methods}

A quantitative and descriptive, cross-sectional survey design was used to assess Addis Ababa's healthcare professionals' physical activity counseling and prescription practice for chronic (non-communicable) diseases in hospital setting from December 2015 to April 2016.

\subsection{Sample and Sampling Procedure}

Based on information from Addis Ababa city health bureau, there are 12 government hospitals in Addis Ababa. Seven sample hospitals were selected randomly based on the inclusion and exclusion criteria.

Inclusion criteria: Participants had to meet the following criteria were included in the study: a registered medical doctors, nurses and physiotherapist, male or female, currently working in the sample hospitals and any age. Hospitals that give services related to: Cardiovascular diseases, Diabetic type 2 and Chronic respiratory diseases were included.

Exclusion Criteria: Participants who met the following criteria were excluded from the study: healthcare professionals still busy with their community service, If the participants are not voluntary and during the study time hospitals were not give services related to cardiovascular diseases, diabetes type 2 and chronic respiratory diseases

Sample Size: The sample size was determined by using the formula for estimating a single population proportion. Sample size was calculated by taking the proportion of physical activity prescription/counseling which is $50 \%$ on healthcare professionals (medical doctors, nurses and physiotherapist) for chronic disease with 95\% confidence level, $5 \%$ margin of error to get an optimum sample size that allowed the study to look into various aspect of physical activity prescription/ counseling among healthcare professionals. Based on the above assumptions, the formula is as follows ( Krejcie and Morgan, 1970):

$\mathrm{s}=\mathrm{X}^{2} \mathrm{NP}(1-\mathrm{P}) \div \mathrm{d}^{2}(\mathrm{~N}-1)+\mathrm{X}^{2} \mathrm{P}(1-\mathrm{P})$

$\mathrm{s}=$ required sample size

$\mathrm{X}^{2}=$ the table value of chi-square for 1 degree of freedom at the desired confidence level

(3.841)---o-- $1.96 \times 1.96=3.8416$

$\mathrm{N}=$ the population size

$\mathrm{P}=$ the population proportion (assumed to be .50 since this would provide the maximum

sample size).

$\mathrm{d}=$ the degree of accuracy expressed as a proportion (.05).

Based on this formula the sample would be 384 , assume $85 \%$ will be return rate, then add $15 \%$, the total sample will be 442 . 


\subsection{Measures and Data Collection Instruments}

Demographic information including physician' sex, age, years of practice, profession and the key variables will collect through questionnaire. Practices of healthcare professionals about physical activity prescription/counseling for their patients were measured by using a scale employed questionnaire of physical activity in the National Family Physician Workforce Survey of Canada (NFWSC; 2010). This scale contains five items measuring the frequency with which healthcare professionals: 1) ask patients about their physical activity, 2) offer verbal prescription, 3) offer written prescription, 4) conduct fitness evaluations, and 5) refer patients to other professionals for fitness assessment. Answers were anchored on a five-point scale ranging from Never $=1$ to Always $=5$.

In addition to this scale, we added 3 additional items in the form of this scale: 1) counsel physical activity for the purpose of preventing chronic diseases, (2) counsel physical activity for the purpose of treating chronic diseases and (3) discuss physical activity with your patients. To increase the validity and reliability of the instruments, after adapting the questionnaires: first evaluated by experts (one nurse, two physicians, one physiotherapist and three sports science professionals). Then based on the feedback the final questionnaire was prepared for pilot study and Second the pilot study was conducted on one hospital and two clinics which are not included in the main sample.

\section{Results}

A total of 442 healthcare professionals from 7 government hospitals in Addis Ababa city were participated in the study. From these hospitals 387 healthcare professionals (physicians $=135,34.9 \%$, nurses $=$ $218,56.3 \%$ and physiotherapist $=34,8.8 \%$ ) were completed the questionnaire.

\subsection{Physical Activity Prescription/Counseling Practices among HCPs}

\section{Figure 3.1: Healthcare professionals' PA prescription/counseling practices}

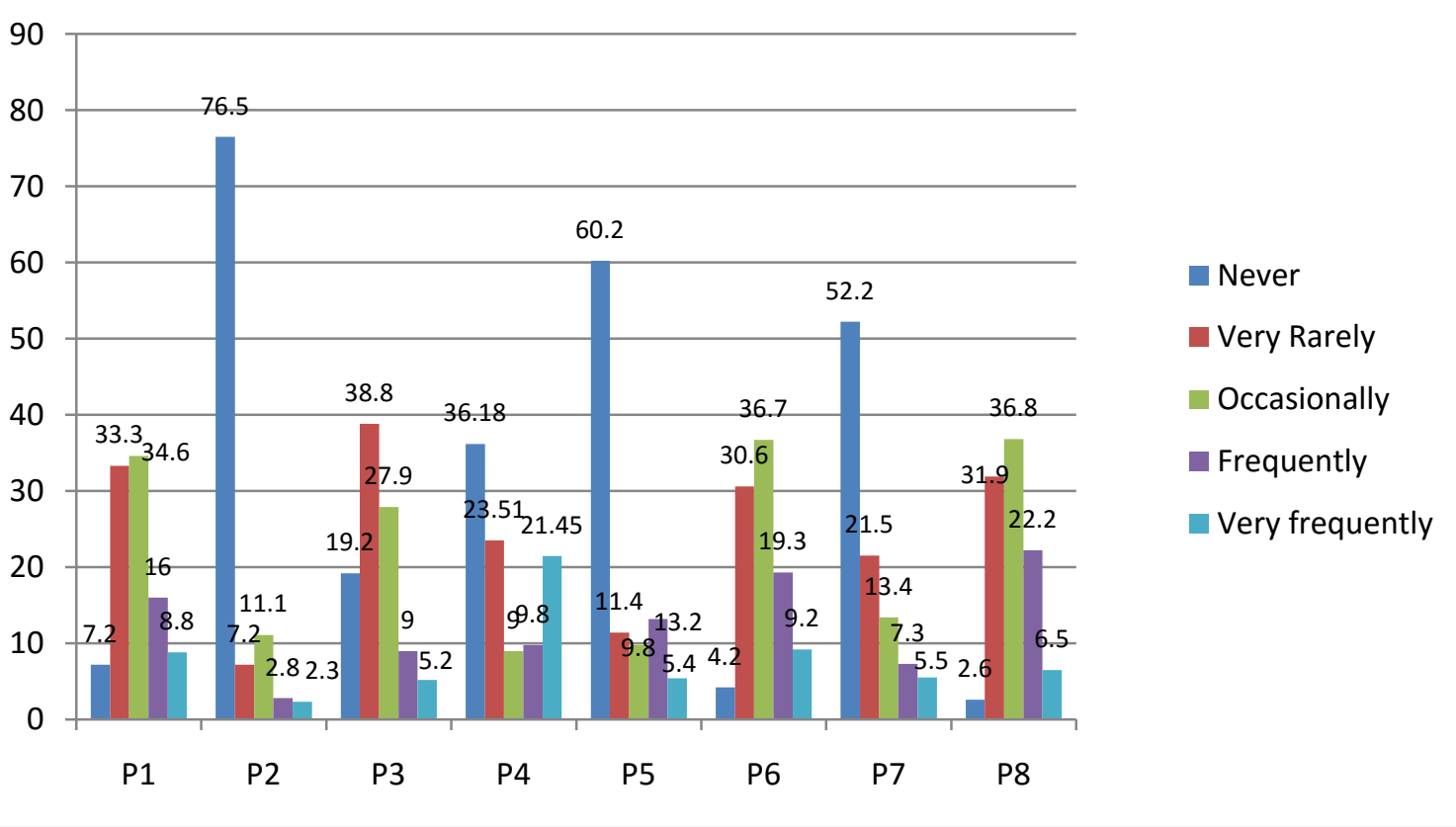

\section{Key Note:}

$\mathrm{p} 1=$ Ask patients about their physical activity levels

$\mathrm{P} 2=$ Assess patient fitness as part of a physical exam or through a fitness test.

P3 = Refer patients to other professionals for fitness assessment or appraisal

P4 = Provide patients with verbal directions for a physical activity program

P5 = Provide patients with written directions for a physical activity program

P6 = Counsel PA (verbal or written prescription) for the purpose of preventing chronic diseases

P7 = Counsel PA (verbal or written prescription) for the purpose of treating chronic diseases

P8 = Discussing about PA with patients

Healthcare professionals practice regarding to asking their patients about physical activity levels reported that: $7.1 \%$ of them never ask their patients about physical activity level, the majority of healthcare 
professionals ask very rarely and occasionally $(33.2 \%, 34.8 \%$ respectively). In other hand few number of HCPs ask frequently and very frequently ( $16.2 \%$ and $8.6 \%$ respectively) their patients about their PA level (PAL).

The reports of assessing patients' fitness during medical exam as part of a physical exam by healthcare professionals in hospital setting shows that: the majority of healthcare professionals (76.2\%) never assess patients' fitness as part of a physical exam or through fitness test, $7.4 \%$ assess patients PAL very rarely, $11.1 \%$ of HCPs occasionally make physical fitness assessment, only about $(5.3 \%=$ frequently + very frequently $)$ indicates that make physical fitness assessment for their patients as a physical exam in their regular practice.

The above graph (Figure 3.1) shows that: $19.1 \%$ of HCPs never refer their patients to fitness professionals, $38.6 \%$ make refers very rarely, $27.9 \%$ make refers for their patients occasionally, $9.1 \%$ of HCPs were provide refer to fitness professional frequently for their patients and $5.2 \%$ very frequently. Here, most of healthcare professionals were provided refers for their patient to fitness professionals very rarely and occasionally, in other way only few number of HCPs were given refers to their patients to fitness professionals frequently.

Regarding to verbal prescription of PA for patients with chronic diseases, about $36.2 \%$ of HCPs never prescribe PA verbally, $23.5 \%$ provide verbal prescription very rarely, $9 \%$ provide verbal prescription occasionally, $9.8 \%$ provide verbal prescription frequently, and $21.4 \%$ provide verbal prescription very frequently. Of 387 participants who prescribed PA to their patients, more than half $(60.2 \%)$ of healthcare professionals reported that, they never provide written prescription of PA to their patients, $11.4 \%$ provide written prescription very rarely, $9.8 \%$ provide written prescription occasionally, $13.2 \%$ provide written prescription frequently and $5.4 \%$ of HCPs provide written prescription very frequently. The majority of healthcare professionals were never provided written physical activity prescription to their patients.

The prevalence of PA counseling for the purpose of preventing chronic diseases by healthcare professionals shows that $4.2 \%$ of HCPs never counsel their patients about PA to prevent chronic diseases, 30.6 $\%$ of HCPs very rarely counsel PA for the purpose of preventing chronic diseases, $36.7 \%$ counsel PA occasionally to their patients for the purpose of preventing chronic diseases, $19.3 \%$ of HCPs frequently provide PA counseling to their patients for the purpose of preventing chronic diseases and only $9.2 \%$ of HCPs very frequently provide PA counseling for the purpose of preventing chronic diseases. The results of counseling PA for the purpose of treating chronic diseases by healthcare professionals reports that more than half $(52.2 \%)$ never counsel physical activity for the purpose of treating/managing chronic diseases, $21.5 \%$ counsel PA very rarely, $13.4 \%$ counsel PA occasionally, $7.3 \%$ of HCPs counsel PA frequently and only $5.5 \%$ of healthcare professionals provide physical activity counseling to their patients for the purpose of managing/treating chronic diseases.

Regarding to the practice of healthcare professionals on routinely counseling physical activity to accomulate 30 minutes of moderate intensity on most of days of the week indicates that most of HCPs $(84.62 \%$ ) does not routinely counsel to accomulate 30 mintes of moderate intensity PA on most days of the week and only few $(15.38 \%)$ of HCPs routinely counsel PA accomulate 30 minutes of moderate intensity on most days of the week.

Personal physical activity level was positively and significantly correlated with physical activity prescription/counseling practices $\left(\mathrm{r}_{\mathrm{s}}=.17, \mathrm{p}=.00\right)$. That means the more physically active the participants, the more frequently prescribe/counsel physical activity to their patients.

To show the differences among healthcare professionals regarding to physical activity prescription/counseling practices, the Kruskal-Wallis Test (the Kruskal-Wallis H Test) was applied. Therefore the Kruskal-Wallis Test indicates that there were a significant difference among the three groups of healthcare professionals $\left(\chi^{2}=147.85, \mathrm{df}=2, \mathrm{p}=.000\right)$. Specifically physiotherapists had the highest physical activity prescription/counseling scores than the other professions again nurses had less mean rank score than the others. The mean rank score of the three groups were physiotherapists (mean rank $=364.31$ ), physicians (mean rank = 236.58) and nurses (mean rank $=141.07$ ).

Significant difference were found between male $(n=177)$ and female $(n=210)$ healthcare professionals regarding to practices of physical activity prescription/counseling $(\mathrm{t}=6.03, \mathrm{df}=385, \mathrm{p}=.000)$. Interestingly, male healthcare professionals significantly more frequently prescribed/counseled physical activity to their patients than female healthcare professionals ( $\mathrm{MD}=.42$ ).

\section{Discussion}

Regarding to physical activity prescription/counseling, there is one study conducted on physical activity promotion in Mexican health care setting (Galaviz K.I. et al, 2015) show that approximately $48 \%$ of physicians reported that they always ask patients about their physical activity levels, 33\% provide verbal physical activity prescription, $6 \%$ provide written physical activity prescription, $4 \%$ assess patient fitness, and $8 \%$ indicated that they always refer patients to other fitness professionals. Regarding to referring patients to other fitness professionals, about $36.5 \%$ of general practitioners refer their patients to another specific local activity or sports club (Leemrijse et al, 2015). One study in south India also show that (42.5\%) doctors reported 
that they always 'asked' their patients about their current PA levels and $46.6 \%$ doctors always gave 'verbal advice'. One-fourth of the doctors (25.3\%) 'always asked and advised' their patients regarding PA ( Patra L, et al., 2015). One-third (32\%) of the healthcare professionals reported that they never or rarely discuss with patients about physical activity (Leemrijse et al, 2015). In line with this our study revealed that $24.8 \%$ of healthcare professionals ask (frequently + very frequently) their patients about their physical activity level, 5.3 $\%$ very frequently assess patients fitness as physical exam, $5.2 \%$ very frequently refer their patients to fitness professionals, $21.4 \%$ very frequently provide verbal physical activity prescription/counseling, only $5.2 \%$ very frequently provide written physical activity prescription, $9.2 \%$ counseling physical activity for the purpose of preventing chronic diseases, $5.5 \%$ counseling PA for the purpose of managing chronic diseases and $28.7 \%$ ( frequently + very frequently) discuss about physical activity with their patients.

In other study conducted on physical activity counseling and prescription among Canadian primary care physicians investigate that (Petrella, Lattanzio and Overend, 2007): $85 \%$ of respondents reported asking patients about their physical activity levels, whereas only $26.2 \%$ assessed patient fitness as part of a physical examination or through a fitness test and only $10.9 \%$ referred patients to others for fitness assessment or appraisal. Most physicians (69.8\%) reported using verbal counseling to promote physical activity, whereas only $15.8 \%$ used written prescriptions for a physical activity promotion program. Those the above studies show that healthcare providers have moderate practice regarding to asking their patients about their physical activity level. But they were not practiced frequently about assessing patients fitness, refer patients to fitness professionals, provide written physical activity prescription/counseling. Similarly our study investigation indicates that healthcare providers' physical activity prescription/counseling practices were less frequent (5.3\% of HCPs very frequently assess patients fitness as physical exam, $5.2 \%$ very frequently refer their patients to fitness professionals, only $5.4 \%$ very frequently provide written physical activity prescription, $9.2 \%$ counseling physical activity for the purpose of preventing chronic diseases, $5.5 \%$ counseling PA for the purpose of managing chronic diseases). In general previous study (in Mexico and Canada) show that most of HCPs ask their patients about their physical activity level (Galaviz K.I. et al, 2015, Petrella, Lattanzio and Overend, 2007).

Regarding to physical activity prescription practices of male and female healthcare provider responded differently, men more frequently assessed fitness than did women, whereas women more frequently asked and provided verbal and written prescription of physical activity for their patients (Petrella, Lattanzio and Overend, 2007). In line with this finding our investigation reported that there was a significant difference between male and female healthcare professionals in their practice. Generally our research finding reported that male healthcare professionals more frequently prescribed/counseled physical activity to their patients than female healthcare professionals in their practice.

Associations between healthcare professionals' physical activity level and their practices of PA prescription/counseling to their patients: our research findings indicate that HCPs' physical activity level was positively and significantly correlated with physical activity prescription/counseling practices $\left(\mathrm{r}_{\mathrm{s}}=.17, \mathrm{p}=.00\right)$. That means the more physically active the participants, the more frequently prescribe/counsel physical activity to their patients. In line with our results other study revealed medical students with higher PA levels agreed with the statements that they would be able to provide better physical activity counseling/prescription if they adhered to exercised and stayed physically active or fit (Holtz et al, 2013, Stanford et al, 2014 and Banday et al, 2015)). Personally active' healthcare professionals promoted PA more frequently and perceived it to be more important than inactive healthcare professionals (Ribera, McKenna and Riddoch, 2005)

In other research also physicians who perform aerobic exercise regularly are more likely to counsel their patients on the benefits of these exercises, as are physicians who perform strength training (Abramson et al, 2000). The medical journal of Assessment of Physical Activity (Counseling) at Primary Health Care (Ahmed S. A ljaberi, 2014) reported that $42 \%$ were physically active. A significant relation was found between physical activity counseling practices and physicians' physical activity level.

Regarding to the difference of healthcare professionals' PA prescription/counseling practices, our research revealed that differences were found among healthcare professionals (physicians, physiotherapist and nurses). The Kruskal-Wallis Test indicates that there were a significant difference among the three groups of healthcare professionals $\left(\chi^{2}=147.85, \mathrm{df}=2, \mathrm{p}=.000\right)$. Among the group healthcare professionals physiotherapists had highest physical activity prescription/counseling practices than other professions.

In general the role of health care providers ( nurses, physicians, physiotherapist etc.) have great value for counseling physical activity in hospitals for prevention and treatment of chronic disease. But some studies show that they are not doing match on physical activity prescription and counseling. "potential" health gain if physicians successfully prescribed physical activity (BMJ,2015). Doctors are well positioned to provide physical activity (PA) counseling to patients. They are a respected source of health-related information and can provide continuing preventive counseling feedback and follow-up; they may have ethical obligations to prescribe PA (Lobelo F., Duperly J. and Frank E., 2008). But healthcare professionals not frequently 
counsel/prescribe physical activity for their patients, why they did not counsel physical activity, they have different reasons some of these lack of time, lack of knowledge, lack of motivation form the patients to perform the recommended physical activity, lack of guidelines and instruction to counsel physical activity in the work place, give low priority to PA etc.

\section{Conclusion}

This is the first study assessing the self-reported PA counseling practices of HCPs. The results of this study indicate a desire to provide PA prescription/counseling. Ethiopian HCPs can play a match greater role on preventing and managing non-communicable diseases through PA counseling /prescription. Despite the low rate of asking patients about PA, assess patient fitness as part of a physical exam or through a fitness test, refer patients to other professionals for fitness assessment or appraisal, provide patients with verbal directions for a PA program, provide patients with written PA prescription, counsel PA (verbal or written prescription) for the purpose of preventing and treating chronic diseases the need for improved implementation and establish strategies to increases HCPs' practice of PA prescription/counseling in hospital setting.

There were a significant difference among the three groups of healthcare professionals. Specifically physiotherapists had the highest physical activity prescription/counseling practices than the other professions. The more physically active the participants, the more frequently prescribe/counsel physical activity to their patients. Regarding to physical activity prescription practices of male and female HCPs responded differently, men more frequently prescribed/counseled PA than did female.

\section{Acknowledgements}

The authors would like to thank Addis Ababa's city administrative health bureau, physicians, physiotherapists and nurses who were participated in the study and Debremarkos university for the financial support.

\section{References}

[1]. Daley A. Exercise and depression: A review of reviews. J Clin Psychol Med Settings (2008) 15:140-147

[2]. American College of Sports Medicine (ACSM 2009).AMCSM's Guideline for Exercise Testing and Prescription, eight edition, ACSM 2009.

[3]. World Health Organization. (2010). Global Recommendations on Physical Activity for Health. Geneva: WHO.

[4]. Lee, I. M., Shiroma, E. J., Lobelo, F., Puska, P., Blair, S. N., \& Katzmarzyk, P. T. (2012). Effect of physical inactivity on major non-communicable diseases worldwide: an analysis of burden of disease and life expectancy. Lancet, 380(9838):219-29

[5]. World Health Organization. Global health risks: mortality and burden of disease attributable to selected major risks. Geneva: World Health Organization, 2009.

[6]. Lee IM, Skerrett PJ. Physical activity and all-cause mortality: what is the dose-response relation? Med Sci Sports Exerc 2001;33: S459e71.

[7]. Physical Activity Guidelines Advisory Committee. (2008). Physical Activity Guidelines Advisory Committee Report 2008. Washington, DC: U.S: Department of Health and Human Services

[8]. WHO( 2011). The Growing Danger of Non-Communicable Diseases. Geneva: WHO.

[9]. Lee I, Shiroma EJ, Lobelo F, Puska P, Blair SN, \& Katzmarzyk PT. (2012). Effect of physical inactivity on major noncommunicable diseases worldwide: an analysis of burden of disease and life expectancy. Lancet, 380, 219-229

[10]. Fiuza-Luces, C., Garatachea, N., Berger, N. A., \& Lucia, A. (2013). Exercise is the real polypill. Physiology, 28(5), 330-358

[11]. WHO. (2012). Global Action plan for the prevention and control of Non-communicable diseases 2013-2020 Zero draft. World health Organization.

[12]. Börjesson M. Promotion of physical activity in the hospital setting. Dtsch Z Sportmed. 2013; 64: 162-165.

[13]. Jacobson, D., M., Strohecker, L., Compton, M., T., \& Katz, D., L. (2005). Physical activity counseling in the Adult primary care setting: Position statement of the American College of Preventive Medicine. American Journal of Preventive Medicine, 29(2):158162 .

[14]. Holub, C.K., Elder, J.P., Arredondo, E.M., Barquera, S., Eisenberg, C.M., Sánchez, L.M., Simoes, E.J. et al (2013). Obesity control in Latin American and U.S. Latinos: asystematic review. American Journal of Preventive Medicine, 44(5), $529-537$.

[15]. Meyer, P., Kayser, B., Kossovsky, M.P., Sigaud, P., Carballo, D., Keller, P.F., . . Mach, F. (2010). Stairs instead of elevators at workplace: cardioprotective effects of a pragmatic intervention. European Journal of Cardiovascular Prevention and Rehabilitation, 17(5):569-575.

[16]. Vuori IM. Lavie CJ. and Blair SN(2013). Physical Activity Promotion in the Health Care System. Mayo Clin Proc. ;88(12):1446-61

[17]. Ribera A. P.,McKenna J., Riddoch C. (2005). Attitudes and practices of physicians and nurses regarding physical activity promotion in the Catalan primary health-care system. European Journal of Public Health: 15(6), 569-575

[18]. Abramson, S., Stein, J., Schaufele, M., Frates, E., \& Rogan, S. (2000). Personal exercise habits and counseling practices of primary care physicians: A national survey. Clinical Journal of Sport Medicine, 10(1), 40-48.

[19]. Petrella RJ, Lattanzio CN, Overend TJ (2007). Physical activity counseling and prescription among Canadian primary care physicians. Arch Intern Med; 167:1774-81.

[20]. Lobelo, F., Duperly, J., \& Frank, E (2009). Physical activity habits of doctors and medical students influence their counseling practices. British Journal of Sports Medicine; 43(2), 89-92.

[21]. Elley CR, Kerse N, Arroll B, et al (2003). Effectiveness of counselling patients on physical activity in general practice: cluster randomized controlled trial. BMJ. 2003;326:793.

[22]. Kallings, L., Leijon, M., Hellenius, M -L., \& Ståhle, A. (2008). Physical activity on prescription in primary health care: A follow up of physical activity level and quality of life. Scandinavian Journal of Medicine \& Science in Sports , $18(2) ; 154-161$ 
[23]. Krejcie, R.V., \& Morgan, D.W. (1970). Determining Sample Size for Research Activities. Educational and Psychological Measurement, 30, 607-610

[24]. The College of Family Physicians of Canada (2010). National Family Physician Workforce Survey. The College of Family Physicians of Canada (2010).

[25]. Olofsgård MJ (2009). Physical Activity Counseling and Prescription in Psychiatry: Swedish Mental Health Professionals' Clinical Practices, Attitudes, and Knowledge, George Mason University (Master's thesis)

[26]. Grimstvedt Megan (2011). Physical Activity Counseling Knowledge, Attitudes, and Practices Among Nurse Practitioners and Physician Assistants. Arizona State University, (Dissertation)

[27]. Galaviz KI, Jauregui E, Fabrigar L, Latimer-Cheung A, Lopez y Taylor J, Lévesque L,(2015). Physical activity prescription among Mexican physicians: a structural equation analysis of the theory of planned behaviour. International Journal of Clinical Practice,69(3)375-383

[28]. Leemrijse C.J, de Bakker D.H2, Ooms L. and Veenhof C .(2015). Collaboration of general practitioners and exercise providers in promotion of physical activity a written survey among general practitioners. BMC Family Practice 16:96

[29]. Patra, Lipika; Mini, G. K.; Mathews, Elezebeth; Thankappan, K. R.. (2015) Doctors' self-reported physical activity, their counselling practices and their correlates in urban Trivandrum, South India: should a full-service doctor be a physically active doctor. British Journal of Sports Medicine; (49) 6: 413

[30]. Petrella, R.J., and Wright, C. (2000). An office-based instrument for exercise counselling and prescription in primary care: The Step Test Exercise Prescription (STEP). Archive of Family Medicine, 9, 339-344.

[31]. Holtz KA. Kokotilo KJ. Fitzgerald BE. Frank E. (2013) Exercise behaviour and attitudes among fourth-year medical students at the University of British Columbia. Can Fam Physician;59:e26-32

[32]. Stanford FC, Durkin MW, Stallworth JR, Powell CK, Poston MB et al (2014). Factors that influence physicians' and medical students' confidence in counseling patients about physical activity. J Prim Prev; 35:193-201.

[33]. Banday AH, Want FA, Alris FFA, Alrayes MF, Mohammad J. and Alenzi MJ. (2015). A Cross-sectional Study on the Prevalence of Physical Activity Among Primary HealthCare Physicians in Aljouf Region of Saudi Arabia, Mater Sociomed ; 27(4): 263-266

[34]. Savill Bryn an Murray Andrew (2015). Is General Practice Engaged With Physical Activity Promotion? British Journal of General Practice, September 2015

[35]. Lobelo, F., Duperly, J., \& Frank, E. (2009). Physical activity habits of doctors and medical students influence theircounselling practices. British Journal of Sports Medicine, 43(2), 89-92.

[36]. Petrella, R.J., and Wright, C. (2000). An office-based instrument for exercise counselling and prescription in primary care: The Step Test Exercise Prescription (STEP). Archive of Family Medicine, 9, 339-344.

[37]. Campkin L, \& Doyle-Baker PK. (2015), Exercise counselling and use of exercise professionals by physicians: Findings from a scoping review.Wellspring; July 8, 2015 